\title{
Does strange kinetics imply unusual thermodynamics?
}

\author{
I.M. Sokolov ${ }^{1}$, J. Klafter ${ }^{2}$ and A. Blumen ${ }^{1}$ \\ ${ }^{1}$ Theoretische Polymerphysik, Universität Freiburg, \\ Hermann-Herder-Str. 3, D-79104 Freiburg, Germany \\ ${ }^{2}$ School of Chemistry, Tel Aviv University, Tel Aviv 69978 IsraelN
}

(October 31, 2018)

We introduce a fractional Fokker-Planck equation (FFPE) for Lévy flights in the presence of an external field. The equation is derived within the framework of the subordination of random processes which leads to Lévy flights. It is shown that the coexistence of anomalous transport and a potential displays a regular exponential relaxation towards the Boltzmann equilibrium distribution. The properties of the Lévyflight FFPE derived here are compared with earlier findings for subdiffusive FFPE. The latter is characterized by a nonexponential Mittag-Leffler relaxation to the Boltzmann distribution. In both cases, which describe strange kinetics, the Boltzmann equilibrium is reached and modifications of the Boltzmann thermodynamics are not required.

PACS No.: 05.40.Fb, 05.70.Ln, 05.60.Cd, 02.50.-r

Strange kinetics [1,2] which involves diffusional anomalies, both sub- and superlinear, and nonexponential relaxations is quite wide-spread and has been observed in a broad range of systems [1] 3] 6]. The ubiquity of strange kinetics rests upon generalization of the central limit theorem due to Lévy [7], a generalization that puts heavytailed distributions on a same level of importance as the well-known Gaussian.

Anomalous diffusion in the presence or absence of an external field has been modelled in a number of ways, including fractional Brownian motion [8], generalized diffusion equations [9], continuous time random walk (CTRW) models [10], Langevin and generalized Langevin equations [11] and generalized thermostatics [12]. In particular CTRW has been demonstrated to be a powerful approach in describing subdiffusive as well as superdiffusive processes and in interpreting experimental results. It is not straightforward, however, to incorporate force fields and boundary conditions in this formalism.

An alternative approach to processes which display strange kinetics is based on fractional equations, which are suitable for handling external fields and for considering boundary value problems. In the case of subdiffusion it was realized that the replacement of the local time derivative in the diffusion equation by a fractional operator accounts for memory effects responsible for anomalous behavior [5,13. In the presence of an external field a fractional Fokker-Planck equation FFPE has been introduced [5],13]:

$$
\frac{\partial}{\partial t} P(x, t)=K_{(\alpha) 0} D_{t}^{1-\alpha} \mathcal{L}_{F P} P(x, t)
$$

where $\mathcal{L}_{F P}$ is the Fokker-Planck operator,

$$
\mathcal{L}_{F P}=\frac{\partial^{2}}{\partial x^{2}}-\frac{\partial}{\partial x} \frac{f(x)}{k_{B} T} .
$$

${ }_{0} D_{t}^{1-\alpha}$ is a fractional Riemann-Liouville operator $0<$ $\alpha<1$, and $K_{(\alpha)}$ is a generalized (sub-)diffusion coefficient, having the dimension $\left[K_{(\alpha)}\right]=\left[\mathrm{L}^{2} / \mathrm{t}^{\alpha}\right]$. The force $f(x)$ is related to the external potential $U(x)$ through $f(x)=-d U / d x$, and $k_{B}$ is the Boltzmann constant. The differential operator ${ }_{0} D_{t}^{1-\alpha}$ acting on functions of time is defined through [14]

$$
{ }_{0} D_{t}^{1-\alpha} Z(t)=\frac{1}{\Gamma(\alpha)} \frac{\partial}{\partial t} \int_{0}^{t} d t^{\prime} \frac{Z\left(t^{\prime}\right)}{\left(t-t^{\prime}\right)^{1-\alpha}} .
$$

The FFPE, Eq.(11), has been derived using a KramersMoyal expansion of the CTRW nonlocal equation [13]. The solution of this FFPE is characterized by a subdiffusive behavior and by a nonexponential Mittag-Leffler decay of the single modes. The decoupled structure of Eq.(1i) guarantees that the Boltzmann distribution is attained at equilibrium 5.13.14. We note that the latter is also a property of the regular Fokker-Planck equation corresponding to $\alpha=1$.

Less clear has been the situation for FFPEs which correspond to Lévy spatial flights. Previously proposed equations 2,11] seem not to lead to the Boltzmann distribution, a point whose impact has been overlooked. This might suggest therefore that strange kinetics requires unusual thermodynamics [12]. Here we derive a FFPE for Lévy flights in the presence of an external force. Our starting point is a representation of Lévy flights in terms of a subordination of random processes [15:16. This representation corresponds to processes in which space and time are decoupled and it does not account for Lévy walks [1,, 4 , 10]. Namely, in what follows we obtain a diverging mean-square displacement in the force-free case. The solution of the FFPE which we derive again leads to the Boltzmann distribution in the equilibrium limit, reemphasizing that there is no need to modify conventional thermodynamics in order to obtain strange kinetics. We bring some examples for solving this FFPE for boundary value problems.

As we proceed to show, the corresponding generalization of the Fokker-Planck equation for Lévy flights is:

$$
\frac{\partial}{\partial t} P(x, t)=-K^{(a)}\left(-\mathcal{L}_{F P}\right)^{\alpha} P(x, t),
$$

where the operator $\left(-\mathcal{L}_{F P}\right)^{\alpha}$ is the $\alpha$-th power of the operator $-\mathcal{L}_{F P}=-\partial^{2} / \partial x^{2}+\partial / \partial x\left(f(x) / k_{B} T\right)$ as will 
be derived below, and the corresponding generalized (super-)diffusion coefficient has as dimension $\left[K^{(\alpha)}\right]=$ $\left[\mathrm{L}^{2 \alpha} / \mathrm{t}\right]$.

The CTRWs can be viewed as Markovian random walks on a lattice (with lattice constant $a$ ) given in terms of the number of steps $n$ of the random walker. $P(x, n)$ is a probability distribution function (pdf) of the particles' displacement $x$ after $n$ steps. The number of steps $n$ performed during the time $t$ follows the probability distribution $S(n, t)$, which may include memory effects 177 . The overall displacement during time $t$ is then given by

$$
P(x, t)=\sum_{n=0}^{\infty} P(x, n) S(n, t) .
$$

In the force-free case the pdf $P(x, n)$ corresponds typically to normal diffusion behavior, and thus $\overline{x^{2}} \propto n$. On the other hand, the typical number of steps can grow subor superlinearly in time, so that the overall behavior can be anomalous.

Here we concentrate on the superdiffusive case and assume that the random process $\{n(t)\}$ is characterized by a diverging mean density of events, so that the first moment of the number $n$ of steps does not exist. As a realization of such a process we can take that the numbers of jumps during different time intervals of unit length are independent random variables distributed according to $S(n, 1) \propto n^{-1-\alpha}$. For $t$ large enough the distribution $S(n, t)$ tends then to a stable Lévy-law $L(n ; \alpha, \beta)[15$. Since $n$ is nonnegative, this law is the one-sided extreme distribution for which $\beta=-\alpha(0<\alpha<1)$. If different time intervals $t$ are considered, the distribution $S(n, t)$ scales as

$$
S(n, t)=\frac{1}{t^{1 / \alpha}} L\left(\frac{n}{t^{1 / \alpha}} ; \alpha,-\alpha\right) .
$$

Imagine now a random walker moving under the influence of a weak force $f(x)$. Such a force introduces an asymmetry into the walker's motion, since the probabilities for forward and backward jumps, $w_{+}$and $w_{-}$are now weighed with the corresponding Boltzmann-factors, $w_{+} / w_{-}=\exp \left(f a / k_{B} T\right)$. For small $f$ one can take $w_{+}=1 / 2+f a / 2 k_{B} T$ and $w_{-}=1 / 2-f a / 2 k_{B} T$. Note that the process described in such a way is a Markovian one, and can be characterized by a transition probability

$$
W\left(x, t+\Delta t \mid x^{\prime}, t\right)=\sum_{n=0}^{\infty} P\left(x-x^{\prime}, n\right) S(n, \Delta t) .
$$

For $\Delta t$ in the intermediate range, i.e. large enough to view both $x$ and $n$ as being continuous and to approximate $P(x, n)$ by the Gaussian $P(x, n)=$ $(2 \pi n)^{-1 / 2} \exp \left(-(x-v n)^{2} / 2 a^{2} n\right)$ with $v=f a / 2 k_{B} T$, yet small enough to have the typical displacement small on the scale of change of $f(x)$, one obtains:

$$
\begin{aligned}
& W\left(x, t+\Delta t \mid x^{\prime}, t\right)= \\
= & \int_{0}^{\infty} \frac{1}{\sqrt{2 \pi n}} \exp \left(-\frac{\left(x-x^{\prime}-v n\right)^{2}}{2 a^{2} n}\right) S(n, \Delta t) d n .
\end{aligned}
$$

The overall Markovian process is then governed by the integral Chapman-Kolmogorov equation

$$
P(x, t+\Delta t)=\int W\left(x, t+\Delta t \mid x^{\prime}, t\right) P\left(x^{\prime}, t\right) d x^{\prime} .
$$

Let us concentrate on the long-time, large $x$, behavior of the system and take the force $f$ to be smooth. On such scales we consider the $x$-Fourier-transform of Eq.(9) and get:

$$
\begin{aligned}
& P(k, t+\Delta t)= \\
= & \int_{0}^{\infty} d n \exp \left(-\left(i k v+k^{2}\right) a^{2} n\right) S(n, \Delta t) P\left(k^{\prime}, t\right) .
\end{aligned}
$$

For random processes leading to diffusive behavior, the first moment of the distribution $S(n, \Delta t)$ for small $\Delta t$ exists, so that one can expand for small $k$ the exponential into a power series, getting:

$$
\begin{aligned}
P(k, t+\Delta t) & =\int_{0}^{\infty} d n\left(1-\left(i k v+k^{2}\right) a^{2} n\right) S(n, \Delta t) P(k, t) \\
& =\int_{0}^{\infty} d n\left(1-\left(i k v+k^{2}\right) a^{2}\langle n\rangle_{\Delta t}\right) P(k, t) \\
& =P(k, t)-\left(i k v+k^{2}\right) a^{2}\langle n\rangle_{\Delta t} P(k, t) .
\end{aligned}
$$

(a Kramers-Moyal procedure). For normal diffusive processes one has $\langle n\rangle_{\Delta t} \simeq w \Delta t$, where $w$ is the jumping rate, so that in the continuum limit

$$
\frac{\partial}{\partial t} P(k, t)=-K\left(i k \frac{f}{k_{B} T}+k^{2}\right) P(k, t),
$$

with $K=a^{2} w / 2$ being the diffusion coefficient. In the $x$-representation this is the conventional Fokker-Planck equation (FPE) [18]:

$$
\frac{\partial P(x, t)}{\partial t}=K\left(-\frac{\partial}{\partial x} \frac{f}{k_{B} T}+\frac{\partial^{2}}{\partial x^{2}}\right) P(x, t) .
$$

In the case when $S(n, \Delta t)$ is a Lévy-stable law of index $\alpha, 0<\alpha<1$, the first moment of $n$ diverges, and the series expansion of the exponential, Eq.(12), is not possible. On the other hand for $\alpha<1$ the integral $\phi(k)=\int_{0}^{\infty} \exp (-\kappa \tau) S(\tau, \Delta t) d \tau$ converges for each $\kappa=\xi+i \eta, \operatorname{Re} \xi>0$, and is a stretched-exponential function 115]. For extreme Lévy-stable distributions with $0<\alpha<1$ (those which vanishing identically for negative arguments) one has $\phi(k)=\exp \left(-\kappa^{\alpha}\right)$. Thus, performing the integration in Eq.(10) one gets:

$$
P(k, t+\Delta t)=\exp \left[-K_{(\alpha)}\left(i k \frac{f}{k_{B} T}+k^{2}\right)^{\alpha} \Delta t\right] P\left(k^{\prime}, t\right)
$$

Expanding now the exponential and repeating the steps leading to Eq.(12) we have 


$$
\frac{\partial P(k, t)}{\partial t}=-K_{(\alpha)}\left(i k \frac{f}{k_{B} T}+k^{2}\right)^{\alpha} P(k, t)
$$

Comparing the terms $-\left(i k f / k_{B} T+k^{2}\right)^{\alpha}$ and $-\left(i k f / k_{B} T+k^{2}\right)$ in Eqs. (16) and (13), which represent the corresponding transport operators $\mathcal{L}_{\alpha}$ and $\mathcal{L}_{F P}=\mathcal{L}_{1}$ in Fourier space, we see that they are connected by the relation $\mathcal{L}_{\alpha}=-\left(-\mathcal{L}_{F P}\right)^{\alpha}$. The same relation holds, of course, when one shifts to the $x$-representation:

$$
\frac{\partial P(x, t)}{\partial t}=-K_{(\alpha)}\left(-\mathcal{L}_{F P}\right)^{\alpha} P(x, t),
$$

see note [19]. Note that Eq.(17) differs from the expressions proposed in 11] where either only the second part of the Fokker-Planck operator (a $\Delta$-term) is changed (and corresponds in our notation to $\left.-\left(-\partial^{2} / \partial x^{2}\right)^{\alpha}\right)$ or where a sum of two terms is introduced, so that fractional space derivatives of the orders $\alpha$ and $2 \alpha$ appear. Note that in general $\mathcal{L}_{\alpha}$ can not be decoupled into additive parts responsible separately for drift and for diffusion.

Some important properties of the Lévy-diffusion in the presence of a force field stem from Eq.(17). Since $-\mathcal{L}_{F P}$ and $\mathcal{L}_{\alpha}$ commute with each of their powers, the eigenfunctions of these operators coincide. The corresponding eigenvalues of $\mathcal{L}_{\alpha}$ are those of $-\mathcal{L}_{F P}$ raised to the power of $\alpha$ :

$$
\lambda_{k}^{F F P}=-\left(-\lambda_{k}^{F P}\right)^{\alpha} .
$$

Note that the eigenfunctions of $-\left(i k f / k_{B} T+k^{2}\right)$ and of $-\left(i k f / k_{B} T+k^{2}\right)^{\alpha}$ (describing conventional FPE and an FFPE in an infinite homogeneous system respectively) can be chosen to be the same. Exemplarily, $\exp (i k x)$ is the eigenfunction of free motion in both cases; we denote its eigenvalues by $\lambda_{k}^{F P}$ and $\lambda_{k}^{F F P}$ respectively. Thus, if $\mathcal{L}_{F P}$ has a (nondegenerate) zero eigenvalue, whose eigenfunction corresponds to a stationary state, the same holds for $\mathcal{L}_{\alpha}$. The stationary states of the systems described by FPE and by FFPE therefore coincide. For closed systems (no currents at infinity), the stationary state is that of thermodynamic equilibrium and is given by the Boltzmann distribution. This is a general property of each subordination process, since a state stationary in $t$ is also stationary in $n$.

The solution of FFPEs under the given initial and boundary conditions can be obtained by means of an eigenfunction expansion, as is generally the case for normal and subdiffusive motion [5, 13, 14, 18]. If $\phi_{m}(x)$ are the eigenfunctions of the Fokker-Planck operator, then the solution of FFPE can be expressed as

$$
P(x, t)=\sum_{m} a_{m} \phi_{m}(x) \Phi_{m}(t)
$$

where $\Phi_{m}(t)$ are the corresponding temporal decay forms. Here the difference between sub- and superdiffusive FFPE gets to be evident: in the subdiffusive case $\Phi_{m}(t)$ are solutions of a fractional ordinary differential equation

$$
\frac{d}{d t} \Phi_{m}(t)=K \lambda_{m 0} D_{t}^{1-\alpha} \Phi_{m}(t)
$$

( $\lambda_{n}$ being real and negative). Hence the $\Phi_{m}(t)$ are Mittag-Leffler functions 12 14. On the other hand the superdiffusive FFPE (being of first order in time) leads to

$$
\frac{d}{d t} \Phi_{m}(t)=-K_{(\alpha)}\left(-\lambda_{m}\right)^{\alpha} \Phi_{m}(t)
$$

corresponding to a simple exponential relaxation, $\Phi_{m}(t)=\exp \left(-K_{(\alpha)}\left|\lambda_{m}\right|^{\alpha} t\right)$. Thus, in the case of a discrete spectrum and of real, negative $\lambda_{m}$ the Lévyflight FFPE retains the exponential nature of the relaxation to equilibrium, a behavior typical for normal FPE, so that only the corresponding relaxation times change. For example, the relaxation behavior of a particle in a harmonic potential, $f(x)=-\gamma x$, follows immediately from a standard solution of the FPE [18]: The eigenfunctions can be expressed through those of the Schroedinger equation, and the spectrum consists of a zero eigenvalue, $\lambda_{0}=0$, and of equidistant negative eigenvalues, $\lambda_{n}=-\left(\gamma / k_{B} T\right) n$. Since the spectrum of a Fokker-Planck operator with a harmonic potential is discrete, the relaxation is multiexponential. The equilibrium state of such a system (the eigenfunction corresponding to $\lambda_{0}=0$ ) shows a Boltzmann distribution. The longest relaxation time is given by the first eigenvalue, $\lambda_{1}=-\gamma / k_{B} T$, so that $\tau=\left(k_{B} T / \gamma\right)^{\alpha} / K_{(\alpha)}$.

Another interesting example corresponds to the motion in the absence of a field of a particle in an interval with absorbing boundaries at $x= \pm l$. The eigenfunctions of the Fokker-Planck operator are now the trigonometric functions, $\phi_{m}(x)=\cos [(m+1 / 2) \pi x / l]$, and the corresponding eigenvalues are $\lambda_{m}=-[(m+1 / 2) \pi / l]^{2}$. The eigenvalues of $\mathcal{L}_{\alpha}$ are $\lambda_{m}=-K_{(\alpha)}[(m+1 / 2) \pi / l]^{2 \alpha}$, so that the overall relaxation again follows a multiexponential pattern. The survival probability for a particle initially situated at the middle of the interval, $x=0$, is equal to

$$
\begin{aligned}
P(t) & =\sum_{m=0}^{\infty} \int_{-l}^{l} \cos \left[(m+1 / 2) \frac{\pi}{l} x\right] e^{\lambda_{m} t}= \\
& =\sum_{n=0}^{\infty} \frac{4}{\pi} \frac{(-1)^{n}}{(2 m+1)} e^{-K_{\alpha}[(n+1 / 2) \pi / l]^{2 \alpha} t} .
\end{aligned}
$$

At longer times this decay tends to a simple exponential with the characteristic time $\tau=K_{(\alpha)}^{-1}(l / 2 \pi)^{2 \alpha}$. Note that the $l$-dependence of this characteristic time differs from that encountered in normal diffusion, where $\tau=K^{-1}(l / 2 \pi)^{2}$. In the case $\alpha=1 / 2$ a simple analytical expression holds at all times: $P(t)=$ $\arctan \left[\exp \left(-\frac{\pi}{2} \frac{K_{(1 / 2)}}{l} t\right)\right]$, see Eq. 5.2.4.8 of Ref. [20].

Using a representation of Lévy flights in terms of a subordination of random processes and following the Kramers-Moyal procedure we have derived a fractional 
Fokker-Planck equation for Lévy flights. It has been shown that when the regular Fokker-Planck operator has a discrete spectrum (as happens under appropriate potentials or boundary conditions) anomalous transport results in an exponential relaxation towards an equilibrium distribution. These properties of the Lévy-flight FFPE are compared with earlier findings for subdiffusive FFPE. The latter is characterized by a non-exponential MittagLeffler relaxation. The equilibrium solution corresponds in both cases to the Boltzmann distribution, emphasizing that there is no need to modify conventional thermodynamics in order to obtain strange kinetics.

The authors gratefully acknowledge the support of the German-Israeli foundation (GIF), of the DFG through SFB428 and of the Fonds der Chemischen Industrie.

[1] M.F. Shlesinger, G.M. Zaslavsky and J. Klafter, Nature 363, 31 (1993)

[2] G.M. Zaslavsky, in: Lévy Flights and Related Topics in Physics, Eds. M.F. Shlesinger, G.M. Zaslavsky and U. Frisch (Springer, Berlin, 1995)

[3] J.-P. Bouchaud and A. Georges, Phys. Rep. 195, 127 (1990)

[4] J. Klafter, M.F. Shlesinger and G. Zumofen, Phys. Today 49, 33 (1996)

[5] R. Metzler, E. Barkai, J. Klafter, Phys. Rev. Lett. 82, 3563 (1999)

[6] V.V. Uchaikin and V.M. Zolotarev, Chance and Stability, Stable Distributions and Their Applications (VSP, Utrecht, 1999)

[7] P. Lévy, Théorie de l'addition des variables aléatoires (Gauthier-Villars, Paris, 1937)

[8] B.B. Mandelbrot, The Fractal Geometry of Nature, (Freeman, N.Y.,1983)

[9] H.G.E. Hentschel and I. Procaccia, Phys.Rev.A 29, 1461 (1984)

[10] J. Klafter, A. Blumen and M.F. Shlesinger, Phys.Rev.A, 35, 3081 (1987)

[11] H.C. Fogedby, Phys. Rev. Lett. 73, 2517 (1994); Phys. Rev. E 58, 1690 (1998)

[12] C. Tsallis, S.V.F. Levy, A.M.C. Souza and R. Maynard, Phys. Rev. Lett. 75, 3589 (1995)

[13] E. Barkai, R. Metzler and J. Klafter, Phys. Rev. E 61, 132 (2000)

[14] R. Metzler and J. Klafter, Phys. Rept. 339, 1 (2000)

[15] W. Feller, An Introduction to Probability Theory and Its Applications, J.Willey \& Sons, NY (1971) vol. I and II.

[16] I.M. Sokolov, PRE in press. See preprint condmat/0007496

[17] A. Blumen, J. Klafter and G. Zumofen, in Fractals in Physics, ed. L.Pietronero and E. Tossati (North Holland, Amsterdam, 1986)

[18] H. Risken, The Fokker-Planck Equation, Springer, Berlin (1984)
[19] The fact that the generalized Fokker-Planck operator $\mathcal{L}_{\alpha}$ equals $-\left(-\mathcal{L}_{F P}\right)^{\alpha}$ and not simply to $\mathcal{L}_{F P}^{\alpha}$ is evident. For example, the force-free case $\left(\mathcal{L}_{F P}=\partial^{2} / \partial x^{2}\right)$ with $\alpha=$ $1 / 2$, when recalling that $\partial P / \partial t=\mathcal{L}_{\alpha} P$, has to describe a symmetric Cauchy distribution, $P=t /\left[\pi\left(x^{2}+t^{2}\right)\right]$. This is not a solution of a Liouville equation $\partial P / \partial t=$ $\partial P / \partial x$.

[20] A.P. Prudnikov, Yu.A. Brychkov and O.I. Marichev, Integrals and Series (Russ.) Moscow, Nauka, 1981 